\title{
Commentary: VATS, RATS, stats, and some caveats
}

\author{
Dhruvin H. Hirpara, MD, ${ }^{a}$ and \\ Biniam Kidane, MD, MSc, FRCSC ${ }^{\mathrm{b}, \mathrm{c}, \mathrm{d}}$
}

Postoperative pain is among the most common indications for opioid initiation in more than 300 million patients undergoing surgery annually worldwide. Although opioids play a crucial role in pain management after major surgery, they have important short-term side effects and long-term public health ramifications, including chronic opioid dependency. Thoracic surgical patients in particular have been shown to have the highest rate of new persistent opioid use compared with other surgical cohorts.

Randomized data demonstrate that video-assisted thoracic surgery (VATS) results in reduced early postoperative pain compared with anterolateral thoracotomy. ${ }^{2,3}$ However, the literature is sparse and lacks direct comparisons of open and minimally invasive techniques with respect to postoperative opioid use. Rajaram and colleagues ${ }^{4}$ present a propensity score-matched study comparing opioid utilization in 16,514 patients undergoing open, VATS, or robotic lobectomy (RL) for primary lung cancer. The authors conclude that RL is associated with reduced postoperative opioid administration during the inpatient period compared with open and VATS approaches.

This study should be lauded for using a large, trusted database to attempt to shed more light on an issue relevant to all thoracic surgeons and the patients we serve. It provides further evidence of the value of minimally invasive surgery, especially in the North American setting.

\footnotetext{
From the a Department of Surgery, University of Toronto, Toronto, Ontario; ${ }^{\mathrm{b}}$ Section of Thoracic Surgery, Department of Surgery, Rady Faculty of Health Sciences, University of Manitoba, Winnipeg, Manitoba; ${ }^{c}$ Department of Community Health Sciences, University of Manitoba, Winnipeg, Manitoba; and ${ }^{\mathrm{d}}$ Research Institute in Oncology and Hematology, Cancer Care Manitoba, Winnipeg, Manitoba, Canada. Disclosures: The authors reported no conflicts of interest.

The Journal policy requires editors and reviewers to disclose conflicts of interest and to decline handling or reviewing manuscripts for which they may have a conflict of interest. The editors and reviewers of this article have no conflicts of interest.

Received for publication June 8, 2020; accepted for publication June 10, 2020; available ahead of print June 30, 2020.

Address for reprints: Biniam Kidane, MD, MSc, FRCSC, GH611-Health Sciences Centre, 820 Sherbrook St, Winnipeg, Manitoba, Canada R3A 1R9 (E-mail: bkidane@hsc.mb.ca).

J Thorac Cardiovasc Surg 2021;162:269-70

$0022-5223 / \$ 36.00$

Copyright (c) 2020 by The American Association for Thoracic Surgery https://doi.org/10.1016/j.jtcvs.2020.06.072
}

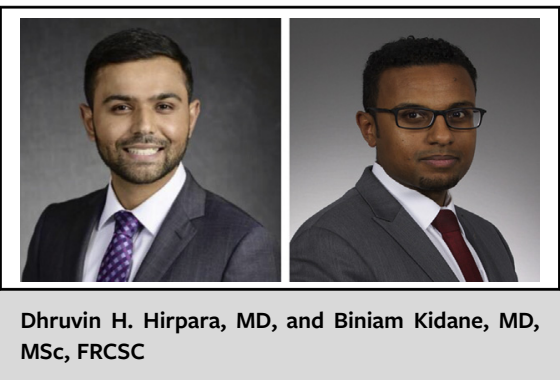

CENTRAL MESSAGE

Minimally invasive thoracic surgery has the potential to improve postoperative opioid utilization, but more robust research into the potential benefits of robotics is needed.

Nonetheless, when it comes to the message regarding the superiority of RL over VATS, caveat lector should be the prevailing watchword. The reader should be mindful of several issues that may color the interpretation of results, conclusions, and inferences. Perhaps most importantly, the authors' relationships with the robotics industry and the study funding provided by Intuitive Surgical must always be kept at the forefront of the reader's mind. This does not necessarily invalidate the study results; it just means that the reader must take an objective view of the extent to which the results are being "spun."

The study findings must also be viewed in light of some methodological issues that may potentially negatively impact the credibility of the authors' conclusions. The authors use propensity score matching (PSM) to control for selection bias and factors that may confound the association of RL with reduced opioid utilization; however, their propensity score did not include tumor size or the need for pleural/rib resection - both of which would be expected to lead to more postoperative pain and opioid use, and are more likely to be approached via open or VATS techniques. ${ }^{5-7}$

A major source of systematic bias is the nonstandardized application of cointerventions. The authors correctly note that their study is limited by the inability to account for differences in application of enhanced recovery after surgery (ERAS) practices among the different groups (open, VATS, and RL). In general, these ERAS cointerventions have been known to be applied in a systematically different and biased way, such that minimally-invasive surgeries are 
more likely than open surgeries to get these cointerventions. Studies in other organ sites (eg, colorectal surgery) have demonstrated that, although minimally invasive surgeries had better perioperative outcomes compared with open surgeries, outcomes were essentially equivalent if ERAS practices were applied equally to both surgical approaches. One of the major contributions of ERAS is in the use of proactive multimodal analgesia, which has the ultimate endpoint of reducing opioid utilization. ${ }^{8}$ In this study, proactive multimodal analgesia was used $8 \%$ more in RL compared with open surgeries and $11 \%$ more in RL compared with VATS. This was in the PSM-matched populations. and there was no adjustment for this. Given the small reported reduction in opioid use with RL, one can reasonably wonder whether these reductions are driven entirely by the use of proactive multimodal analgesia rather than by surgery type. This is especially true for the VATS versus RL comparison, for which the authors report that RL is associated with only $1 \%$ to $4 \%$ less use of opioids despite a $11 \%$ greater use of proactive multimodal analgesia in the RL group.

A final caveat is about the bias introduced by clinician beliefs in a nonrandomized and unblinded study whose outcome is clinician-determined or clinician-driven (here, opioid use). Surgeons tend to prescribe less analgesics (especially opioids) for minimally invasive surgeries for many reasons, not the least of which is a belief that these surgeries are less painful. The less invasive the surgery appears to be, the less opioids are prescribed. Surgeons who perform RL are presumably doing so because they are convinced it is better and less painful; thus, it is not surprising that they would systematically use less opioids for RL cases. It is in essence a self-fulfilling prophecy. This is not necessarily a nefarious phenomenon in and of itself; it just should be kept in mind as a major potential source of unresolved bias. When there is no randomization of intervention or blinding of who is getting the intervention, such beliefs (correct though they may be) systematically bias any clinician-driven outcome.

This study is an important addition to the literature, confirming in a North American population that minimally invasive surgery is associated with reduced opioid utilization. Nonetheless, there are several caveats and unresolved bias that cloud any inferences about the superiority of robotic surgery over VATS on postoperative opioid utilization. In the end, robotic surgery may in fact be the way of the future; however, as we blaze a path towards the future, we must remember that the best way to avoid stumbling into pitfalls is to keep our eyes wide open.

\section{References}

1. Clarke H, Soneji N, Ko D, Yun L, Wijeysundera DN. Rates and risk factors for prolonged opioid use after major surgery: population-based cohort study. BMJ. 2014; 348:g1251.

2. Lim E, Batchelor T, Dunning J, Shackcloth M, Anikin V, Naidu B, et al. Inhospital clinical efficacy, safety and oncologic outcomes from VIOLET: a UK multi-centre RCT of VATS versus open lobectomy for lung cancer. $J$ Thorac Oncol. 2019;14:S6.

3. Bendixen M, Jørgensen OD, Kronborg C, Andersen C, Licht PB. Postoperative pain and quality of life after lobectomy via video-assisted thoracoscopic surgery or anterolateral thoracotomy for early-stage lung cancer: a randomised controlled trial. Lancet Oncol. 2016;17:836-44.

4. Rajaram R, Rice DC, Li Y, Bruera E, Liu E, Song C, et al. Postoperative opioid use after lobectomy for primary lung cancer: a propensity-matched analysis of premier hospital data. J Thorac Cardiovasc Surg. 2021;162:259-68.e4.

5. Santos HT, Lopes AJ, Higa C, Nunes RA, Saito EH. Lung cancer with chest wall invasion: retrospective analysis comparing en-bloc resection and 'resection in bird cage'. J Cardiothorac Surg. 2014;9:57.

6. Homma T, Doki Y, Yamamoto Y, Ojima T, Shimada Y, Kitamura N, et al. Risk factors of neuropathic pain after thoracic surgery. J Thorac Dis. 2018; 10:2898-907.

7. Novellis P, Bottoni E, Voulaz E, Cariboni U, Testori A, Bertolaccini L, et al. Robotic surgery, video-assisted thoracic surgery, and open surgery for early-stage lung cancer: comparison of costs and outcomes at a single institute. $J$ Thorac Dis. 2018;10:790-8.

8. Semenkovich TR, Hudson JL, Subramaniam M, Kozower BD. Enhanced recovery after surgery (ERAS) in thoracic surgery. Semin Thorac Cardiovasc Surg. 2018; 30:342-9. 